\title{
Interference-Aware Radio Resource Allocation for 5G Ultra-Reliable Low-Latency Communication
}

\author{
Hassan Malik, Muhammad Mahtab Alam, Yannick Le Moullec \\ School of Information Technologies \\ Tallinn University of Technology \\ Tallinn, Estonia \\ \{hassan.malik, muhammad.alam, yannick.lemoullec\}@ttu.ee
}

\author{
Qiang Ni \\ School of Computing and Communications \\ Lancaster University \\ Lancaster, UK \\ q.ni@lancaster.ac.uk
}

\begin{abstract}
Ultra-reliable low-latency communication (URLLC) is one of the main challenges faced by future $5 \mathrm{G}$ networks to enable mission critical IoT use-case scenarios. High reliability can be achieved by reducing the requirement of achievable rates, therefore, results in reduced spectral efficiency. Retransmission has been introduced for $5 \mathrm{G}$ or beyond, to achieve reliability with improved spectral efficiency at the cost of increased packet latency. Keeping in mind, the trade-off between reliability and latency, in this paper, we have proposed an interference-aware radio resource (IARR) allocation for uplink transmission by formulating a sum-rate maximization problem. The aim of proposed algorithm is to improve the link quality to achieve high reliability for future $\mathbf{5 G}$ networks resulting in reduced retransmissions and packet latency. To reduce the computation complexity of the maximization problem in achieving the global optimal solution, we propose a progressive interference-aware heuristic solution. The proposed solution is then investigated to evaluate the impact of retransmission and inter-cell interference on the average information rate and latency of the considered multi-cell cellular network. The performance of IARR algorithm is then compared with the conventional round-robin scheduling (RRS). Significant improvement in the link reliability along with the reduction in latency has been observed with IARR algorithm. The results illustrate that the IARR algorithm improves the average rate by $7 \%$ and latency by $10 \%$ compared to RRS.
\end{abstract}

Index Terms-5G new radio (5G-NR), Ultra-reliable lowlatency communication (URLLC), Resource allocation, Retransmission, System-level evaluation.

\section{INTRODUCTION}

The recent evolution of 5 th generation new radio (5GNR) standard will support a wide range of services and application. The focus is not only on enhancing the mobile broadband service but also enabling infrastructure for real-time and mission-critical services. $5 \mathrm{G}$ will target to support ultrareliable low-latency communication (URLLC) where small packets transmission (i.e., 32 bytes) become possible within $1 \mathrm{~ms}$ latency and $99.99 \%$ packet reception rate [1]. Support for such stringent requirements will enable a range of new business models and services for the Internet of Things (IoT) use-cases such as automated factories and vehicular communications [2], [3], and importantly will enable mobile network operators, software companies, original equipment manufacturers (OEMs) and entertainment providers to open valuable new revenue streams.
However, to meet such challenging requirements, new technical innovations need to be adopted. In this regard, 3rd Generation Partnership Project (3GPP) has accepted some new techniques to be considered for URLLC communication, particularly for low-layer functionalities such as modulation, coding, orthogonal frequency-division multiplexing (OFDM) numerology, link adaptation, hybrid repeat request etc [4], [5]. Particularly, to achieve high reliability, retransmission is introduced in Long Term Evolution (LTE) and planned for 5G. No doubt, retransmission improve the link reliability but at the cost of increased packet latency. To achieve, the target requirements of URLLC, it has been found that the main obstacle is the inter-cell interference. Inter-cell interference directly degrades the link reliability which in turn increase the latency due to retransmission [6].

As mentioned, most of the efforts so far have been dedicated to low-layer functions and little has been done on upper-layer functionalities i.e., inter-cell interference, radio resource management, etc. For example, in [7], three multi-cell coordinated techniques in an indoor scenario has been evaluated namely, single frequency network, narrowband muting and macro diversity with soft combining. It has been shown that inter-cell coordination improves the link reliability. However, inter-cell coordination techniques reduce the spectral efficiency by restricting the transmission of certain resources. As it is expected that URLLC has been given a small portion of bandwidth similarly to narrowband IoT, applying such techniques might not be a feasible solution. Furthermore, in [8], different micro and macroscopic diversity techniques along with interference management scheme to achieve the required outage probability in the downlink for URLLC was investigated in a realistic network. Similarly, in [9], the analytical tools to characterize the delay and packet loss components for URLLC have been identified and challenges in resource allocation have been presented.

This paper presents an interference-aware radio resource (IARR) allocation for uplink users with an aim to improve the signal-to-interference-ratio (SINR) and reduce the retransmissions. The rate maximization problem is formulated considering the required quality of service (QoS) constraints. Due to the complexity of finding the globally optimum solution, we provide a heuristic algorithm based on two steps namely; 
resource block allocation and power allocation. Furthermore, the performance of the proposed algorithm is then compared with round-robin scheduling (RRS).

The rest of the paper is structured as follows. Section II present the problem formulation along with the details of a scenario under consideration. Section III discuss the proposed IARR algorithm in detail. The evaluation of IARR algorithm in terms of information rate and latency is presented in Section IV along with the comparison with RRS and simulation parameters. Finally, Section V presents briefly the conclusion of the paper.

\section{Rate Maximization Problem Formulation}

As mentioned in Section I, to improve the system reliability and reduce the latency at the same time, one of the keys is to reduce the retransmission by improving the SINR of the communication link. The number of retransmissions is evaluated during the link adaptation process along with the modulation and coding scheme (MCS) selection based on the maximum coupling loss (MCL) as provided by 3GPP [10]. Whereas, MCL of the user is given as follows [11]:

$$
\begin{aligned}
\mathrm{MCL} & =\text { Transmit power }+174-\text { Noise figure } \\
& -10 \log _{10}(\text { Bandwidth })-\mathrm{SNR}
\end{aligned}
$$

To improve the link reliability and reduce the retransmissions, it is crucial to improve the SINR of user in the presence of inter-cell interference with an appropriate resource allocation scheme. Therefore, in this paper, a multi-cell cellular network, where each cell comprises of three sectors, is studied and the focus is on the uplink transmission where user expect inter-cell interference from the neighboring cell uplink users in the same resource as shown in Figure 1. This paper assumes a set of base stations $\mathcal{B}=\{1, \ldots, B\}$ that communicates over OFDM to multiple user terminals. The total transmission frame duration is considered to be $10 \mathrm{~ms}$, which consists of set mini time-slots $\mathcal{T}=\{1, \ldots, T\}$ consisting of $1 \mathrm{~ms}$ and one physical resource block (PRB) of $180 \mathrm{kHz}$. We assume that there are a set of $\mathcal{M}=\{1, \ldots, M\}$ active users in each cell. The SINR of the $m^{\text {th }}$ user of cell $b$ in time-slot $t$ can be given by [12]:

$$
\begin{array}{r}
\gamma_{m, b, t}=\frac{P_{m, b, t} \Gamma_{m, b, t}}{N_{o}+\sum_{k \in B} P_{m, k, t} \Gamma_{m, k, t}^{b}} \\
\forall m \in \mathcal{M}, b \in \mathcal{B}, t \in \mathcal{T}
\end{array}
$$

where $P_{m, b, t}$ denotes the transmission power of $m^{\text {th }}$ user of cell $b$ in time-slot $t, \Gamma_{m, b, t}$ is the channel gain between base station $b$ and $m^{\text {th }}$ user in time-slot $t, N_{o}$ is the noise power spectral density per PRB. $P_{m, k, t}$ is the transmission power of $m^{\text {th }}$ user of neighboring cell $k$ in time-slot $t$ and $\Gamma_{m, k, t}^{b}$ is the channel gain between $m^{\text {th }}$ user of neighboring cell $k$ in timeslot $t$. Using the Shannon-Hartley Theorem, the achievable rate of user can be calculated as follows:

$$
R_{m, b, t}=\log _{2}\left(1+\gamma_{m, b, t}\right)
$$

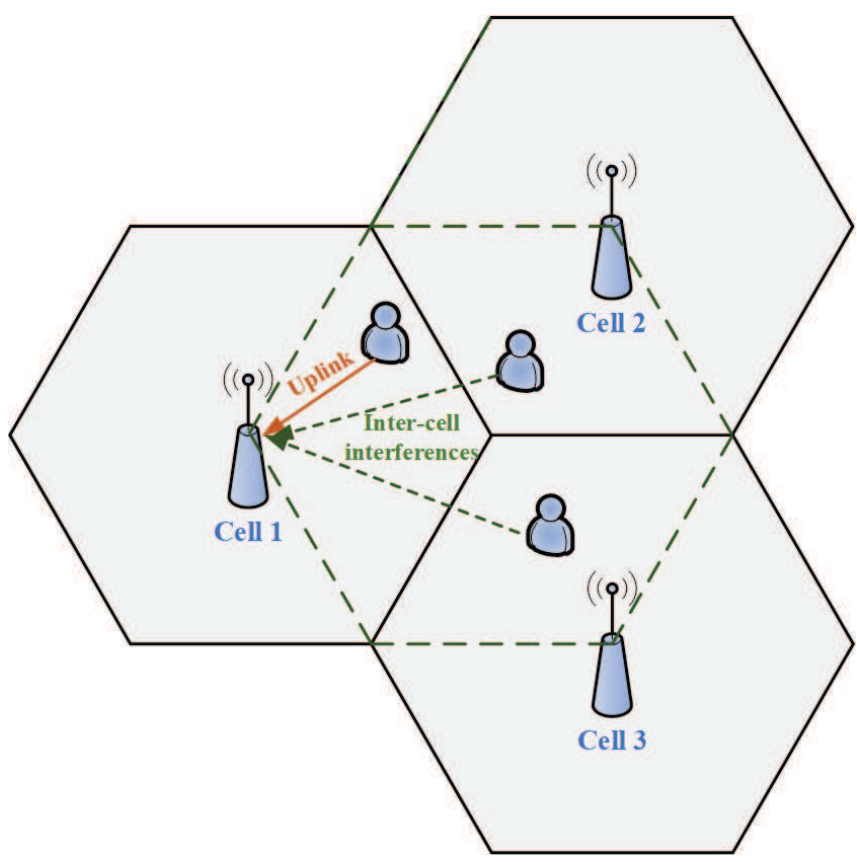

Fig. 1. Multi-cell cellular network with three sectors and the potential intercell interference expected from neighboring sector users sharing the same radio resource.

\section{A. Interference Allowance}

The minimum overall data rate demand of each $m^{\text {th }}$ user of cell $b$ in time-slot $t$ can be mapped to the minimum data rate demand, $R_{m, b, t}^{\min }$, in each time-slot $t$, allocated to that specific user. Furthermore, the minimum data rate demand in timeslot can be translate into a specific minimum required SINR, denoted by $\gamma_{m, b, t}^{r e q}$. With the information of minimum required SINR, the maximum interference power $\Phi_{m, b, t}^{\max }$ that user can tolerate in time-slot $t$ from the neighboring cell user to obtain this rate threshold can be calculated using Eq.(2) as:

$$
\Phi_{m, b, t}^{\max }=\frac{P_{m, b, t} \Gamma_{m, b, t}}{\gamma_{m, b, t}^{r e q}}-N_{o}
$$

If the potential channel gain from any $m^{\text {th }}$ user of neighboring cell $k$ in time-slot $t$ is denoted by $\Gamma_{m, b, t}^{(k)}$ to the base station $b$, the total interference caused to the $m^{\text {th }}$ user of cell $b$ in time-slot $t$ can be given by:

$$
\begin{aligned}
\Phi_{m, b, t}^{s u m} & =\sum_{k \in B} P_{m, k, t} \Gamma_{m, b, t}^{k} \\
& =\sum_{k \in B} \eta_{m, k, t}^{b}
\end{aligned}
$$

where $\eta_{m, b, t}^{k} \triangleq P_{m, k, t} \Gamma_{m, b, t}^{k}$ is the interference caused to $m^{\text {th }}$ user of cell $b$ in time-slot $t$. 


\section{B. Rate optimization}

The sum-rate maximization problem with the constraints can be formulated as:

$$
\max _{x_{m, b, t}, P_{m, b, t}} \sum_{m \in \mathcal{M}} \sum_{b \in \mathcal{B}} \sum_{t \in \mathcal{T}} x_{m, b, t} R_{m, b, t}
$$

subject to

$$
\begin{gathered}
x_{m, b, t} \in\{0,1\}, \quad \forall m \in \mathcal{M}, b \in \mathcal{B}, t \in \mathcal{T} \\
\Phi_{m, b, t}^{s u m} \leq \Phi_{m, b, t}^{\max }, \quad \forall m \in \mathcal{M}, b \in \mathcal{B}, t \in \mathcal{T} \\
\sum_{t \in \mathcal{T}} R_{m, b, t} \geq R_{m, b, t}^{\text {min }}, \quad \forall m \in \mathcal{M}, b \in \mathcal{B}, t \in \mathcal{T} \\
\sum_{t \in \mathcal{T}} P_{m, b, t} \leq P_{\max }, \quad \forall m \in \mathcal{M}, b \in \mathcal{B}, t \in \mathcal{T} \\
P_{m, b, t} \geq 0, \quad \forall m \in \mathcal{M}, b \in \mathcal{B}, t \in \mathcal{T}
\end{gathered}
$$

Where $x_{m, b, t}$ is the time-slot allocation index which indicates that PRBs are exclusively allocated to one user in each timeslot to avoid intra-cell interference. Furthermore, $x_{m, b, t}=1$ if time-slot is allocated to a particular user and otherwise, $x_{m, b, t}=0$. Constraint (8) presents the total maximum interference (i.e. inter-cell interference) that a user can tolerate in order to satisfy its minimum rate needs, constraint (9) is the minimum rate requirement of each user. Finally, constraints (10)-(11) indicate the maximum and minimum transmit power of the user.

\section{Proposed Resource Allocation Scheme}

The formulated optimization problem in (6)-(11) cannot be expressed as a convex optimization problem. The reason is the presence of a non-convex set of the binary variable in constraint (7). Furthermore, due to the interference terms in objective function due to (2), it is really hard to solve find the global optimal solution. Therefore, in this paper, the proposed interference-aware resource allocation algorithm heuristically achieve the objective function expressed in problem (6)-(11). No doubt, the proposed algorithm provides the sub-optimal solution, the aim is to reduce the computation complexity to make the solution feasible for practical networks. The proposed heuristic algorithm comprises three-step namely: resource block allocation, power allocation, link adaptation.

\section{A. Time-slot Allocation}

In the first step, the proposed algorithm initially assign the resource blocks to each user based on the rate/SINR required $\left(\gamma_{m, b, t}^{r e q}\right)$ by the user for the uplink transmission. However, to do so and to make solve the problem easily, first we relax the constraint $x_{m, b, t} \in\{0,1\}$ to take any real value between 0 and $1, x_{m, b, t} \in[0,1]$. It has been shown that with this relaxation, the problem becomes convex and has no duality gap [13]. With this relaxation, to maximize the system rate, greedy allocation approach is considered and the user with the best channel gain is selected in each resource block as follows:

$$
x_{m, b, t}=1, \text { ifm }=\underset{m \in M}{\arg \max }\left(\gamma_{m, b, t}\right)
$$

After the initial allocation, based on the channel gain and the required SINR of each user on the allocated resource block, maximum interference $\left(\Phi_{m, b, t}^{\text {sum }}\right)$ that user can tolerate in that time-slot can be estimated using (4). The information of the maximum tolerable interference is then shared with the neighboring cell through X2 interface. This information will help to find the appropriate power level of each user sharing the resource and imposed as a restriction so that both transmissions can achieve their rate requirements.

\section{B. Power Allocation}

With the given resource allocation, the power allocation problem can be simplified as follows:

$$
\begin{array}{r}
\max _{P_{m, b, t}} \sum_{t \in \mathcal{T}} R_{m, b, t} \\
\text { subject to }(8)-(11) \\
\text { where } \\
\gamma_{m, b, t}^{\sim}=\left\{\gamma_{m, b, t}: x_{m, b, t}=1\right\}
\end{array}
$$

At first each user find the transmit power required to satisfy the minimum rate requirement as follows:

$$
\begin{aligned}
\log _{2}\left(1+\frac{P_{m, b, t} \Gamma_{m, b, t}}{N_{o}+\eta_{m, b, t}^{k}}\right) \geq R_{m, b, t}^{\min } \\
\text { where } \\
\eta_{m, b, t}^{k}=\Phi_{m, b, t}^{\max }
\end{aligned}
$$

After some mathematical manipulation,

$$
P_{m, b, t}=\frac{1}{\Gamma_{m, b, t}}\left(2^{R_{m, b, t}^{m i n}}-1\right)\left(N_{o}+\eta_{m, b, t}^{k}\right)
$$

As stated, the transmit power is now computed based on the interference restriction imposed by the neighboring cell users

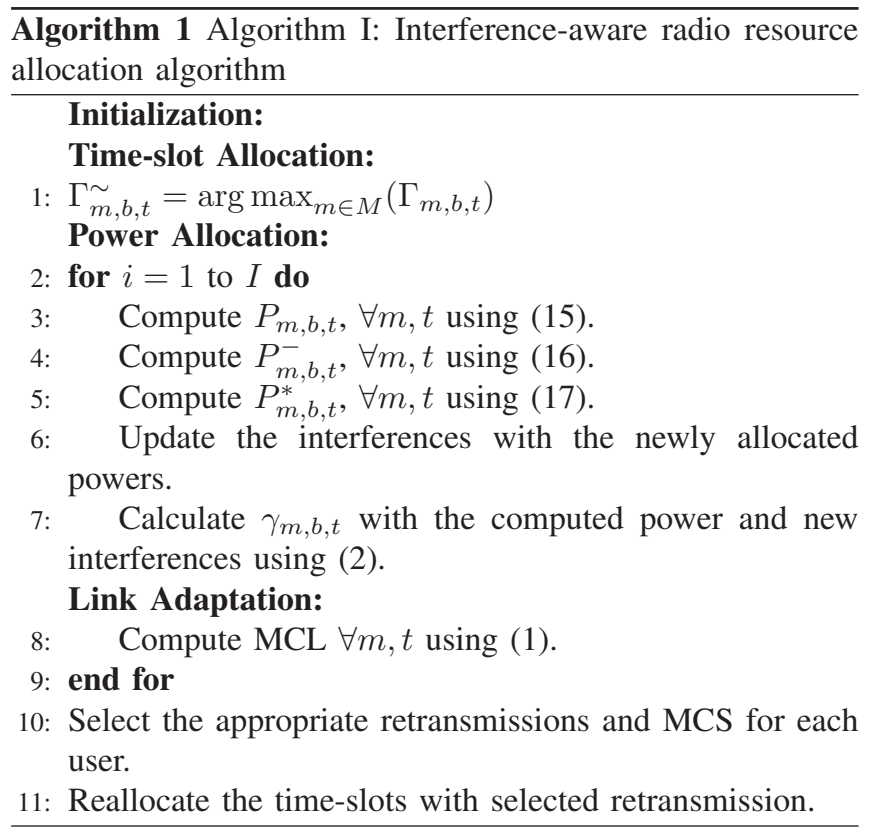


in terms of maximum allowable interference using (5), as follows:

$$
P_{m, b, t}^{-}=\frac{\Phi_{m, k, t}^{\max }}{\Gamma_{m, b, t}^{k}}
$$

Hence, the final constraint transmit power for each user can be calculated as follows:

$$
\begin{array}{r}
P_{m, b, t}^{*}=\left\{\begin{array}{cc}
\min \left(P_{m, b, t}, P_{m, b, t}^{-}, P_{j_{k}, t}^{\max }\right), & \text { if } \mathcal{X} \geq 0 \\
\text { infeasible, } & \text { otherwise }
\end{array}\right. \\
\text { where, } \mathcal{X}=\min \left(P_{m, b, t}, P_{m, b, t}^{-}\right)
\end{array}
$$

\section{Link Adaptation Parameter Selection}

After the power allocation, the respective SINR of each user is determined and the corresponding MCL is calculated based on (1). With the MCL, the retransmission factor is selected along with the MCS. The algorithm then reassigns the time-slots along with the needed retransmissions. The detailed algorithm is listed in Algorithm 1.

\section{Simulation Results}

\section{A. Simulation setup}

For the performance analysis, we have considered a regular hexagonal multi-cell cellular network with three sectors in each cell and an inter-site distance of $500 \mathrm{~m}$. The simulation assumption and parameters are derived from the 3GPP standard [14] as presented in Table I. The total frame transmission is assumed to be $10 \mathrm{~ms}$ with mini-slot of $1 \mathrm{~ms}$. Each minislot consist of one PRB of $180 \mathrm{kHz}$, comprising of 12 subcarriers with $15 \mathrm{kHz}$ subcarrier spacing. Furthermore, the retransmission factor is computed based on MCL for different coverage classes and presented in Figure 2 [11].

The traffic of each user is modeled according to the 3GPP standard [14] annex E, Mobile Autonomous Reporting (MAR) periodic traffic model. the traffic model is characterized as follows:

- Pareto distributed application payload size with alpha having a value of 2.5 and beta with a minimum and maximum value of 20 bytes and 200 bytes, respectively, is considered. As in IoT use-cases, reports are usually not very large in size. We assumed three different packet sizes of 27, 35 and 50 bytes with the percentage of $50 \%, 75 \%$, and $90 \%$. The men size is of 32 bytes. The assumption follows the parameters provided in Nokia evaluation document for NB-IoT [15].

- The arrival time of packets from different users are distributed into various categories with constant interarrival time and device proportion of one day (40\%), two hours $(40 \%)$, one hour $(15 \%)$ and 30 minutes $(5 \%)$ as in [15]. Considering, $52 \mathrm{~K}$ devices per cell and the network of seven cells (each with three sectors), this will lead to 21 cells in total and gives 143 reports per second per network as calculated in [15].

Moreover, in terms of MCS, each user will apply the QPSK and $1 / 3$ code rate on all the transmission. Also, a cyclic
TABLE I

Simulation PARAmeters

\begin{tabular}{|l|l|}
\hline Parameters & Values \\
\hline Cell layout & Hexagonal grid, 3 sectors per cell \\
\hline Frequency band & $900 \mathrm{MHz}$ \\
\hline Inter-site Distance & $500 \mathrm{~m}$ \\
\hline User distribution & Users dropped uniformly in entire cell \\
\hline Base station transmit power & $32 \mathrm{dBm}(3 \mathrm{~dB}$ boosting applied) \\
\hline User transmit power & $23 \mathrm{dBm}$ \\
\hline Pathloss Model & $\mathrm{L}=\mathrm{I}+37.6 \log _{10}(R)$, \\
& $\mathrm{I}=120.9$ for the 900 MHz band \\
& where $R$ in kilometers \\
\hline Shadowing standard deviation & $8 \mathrm{~dB}$ \\
\hline Shadowing Correlation: & $110 \mathrm{~m}$ \\
Distance & 0.5 \\
Between cell sites & 1.0 \\
Between cell sectors & $18 \mathrm{dBi}$ \\
\hline Base station antenna gain & $-4 \mathrm{dBi}$ \\
\hline User antenna gain & $3 \mathrm{~dB}$ \\
\hline Base station cable loss & {$[2040] \mathrm{dB}$} \\
\hline Building penetration loss & $5 \mathrm{~dB}$ \\
\hline Noise figure at base station & $3 \mathrm{~dB}$ \\
\hline Noise figure at user & $-174 \mathrm{dBm} / \mathrm{Hz}$ \\
\hline Noise power spectral density & \\
\hline
\end{tabular}

redundancy check (CRC) of 3 bytes and a compressed header of 29 bytes will be appended with each data transmission as mentioned in [14].

\section{B. Simulation results}

In this subsection, the performance of the proposed algorithm is investigated in both single-cell and multi-cell scenario with Monte-Carlo simulations. The performance evaluation in terms of average information rate and average latency with different penetration losses is presented and discussed in detail. With the simulation parameters presented in Table I, the simulations are rum for uniformly distributed users for 500 iterations.

The results are first generated for average information rate per sector with $25 \mathrm{~dB}$ penetration loss as shown in Figure 3. The results present a comparison of single-cell, multi-cell with

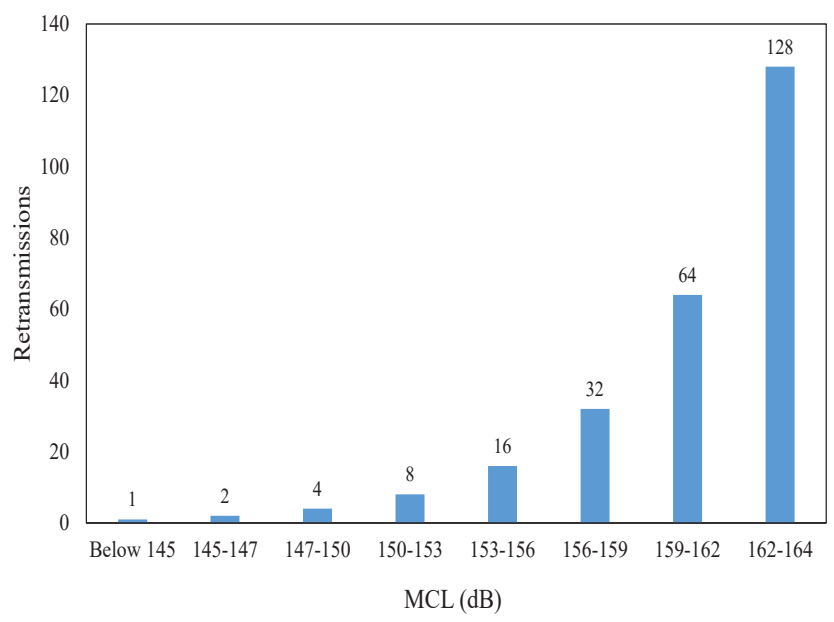

Fig. 2. Coverage classes with number of retransmissions. 


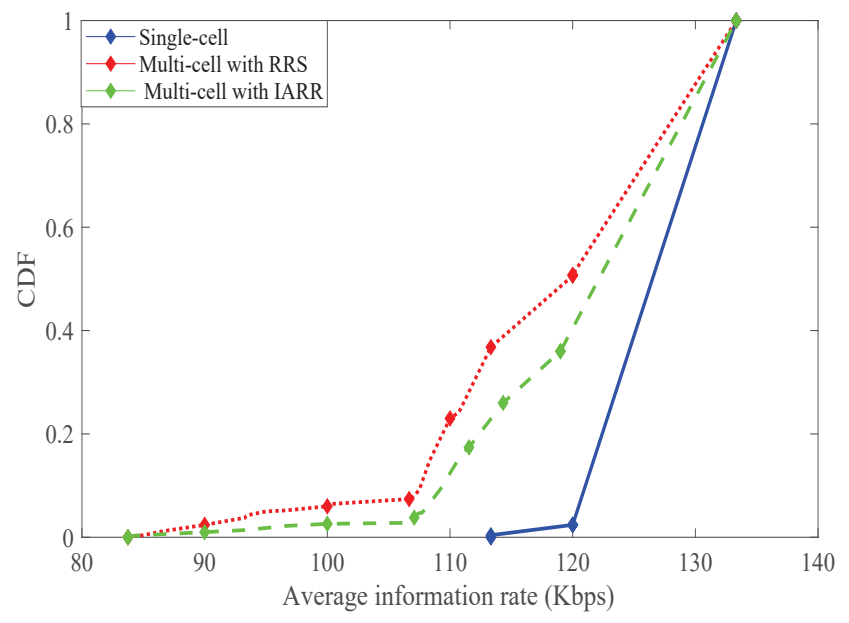

Fig. 3. Average information rate per sector with $25 \mathrm{~dB}$ penetration loss.

RRS and multi-cell with IARR in-terms of average information rate. It can be seen that the impact of inter-cell interference is quite significant in a multi-cell environment. However, the proposed algorithm significantly improves the performance by mitigating the impact of inter-cell interference and improve the link reliability.

Figure 4 present the average information rate of users per sector with different penetration losses. The results show that, at high penetration loss, the impact of inter-cell interference is not significant and the performance is almost the same in most single-cell and multi-cell scenario. At low penetration loss, the inter-cell interference dominates the system. For example, at the penetration loss of $10 \mathrm{~dB}$, the average information rate in single-cell is $133 \mathrm{Kbps}$, whereas, in multi-cell with RRS, the rate drops to $120 \mathrm{Kbps}$. However, the proposed algorithm significantly improves the performance and achieve around $128 \mathrm{Kbps}$, which results in approx. 7\% gain in performance compared to multi-cell with RRS. The proposed algorithm

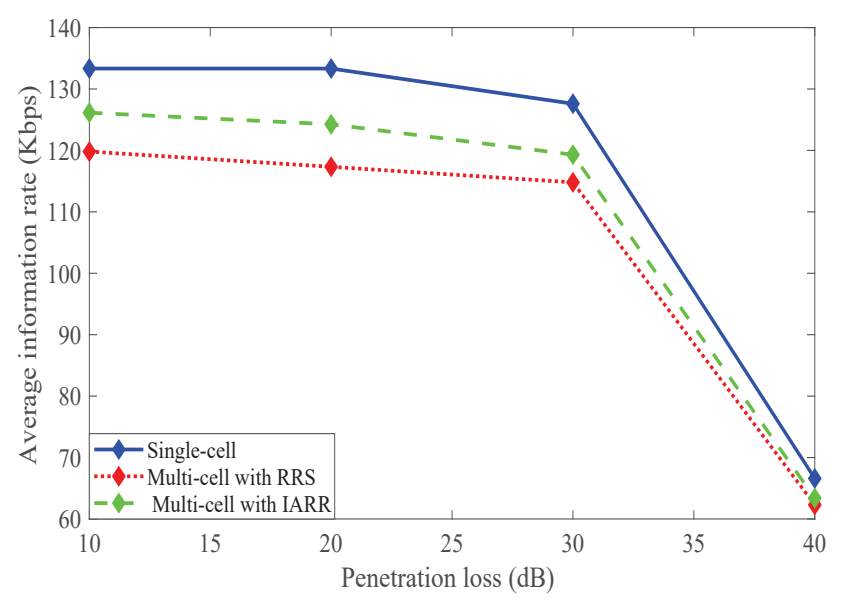

Fig. 4. Impact of penetration loss on average information rate.

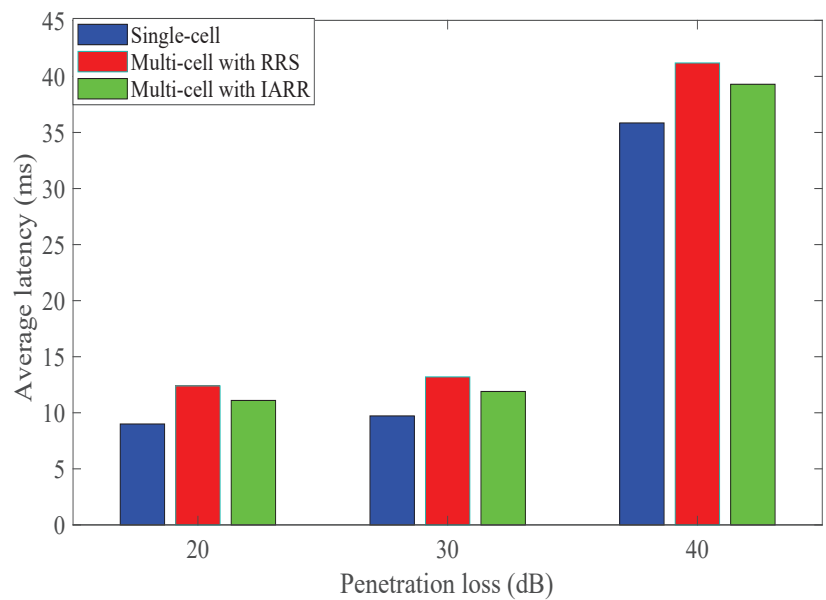

Fig. 5. Impact of penetration loss on average latency

improves the information rate by reducing the impact of intercell interference and improving the link quality, this results in a reduction of retransmissions.

The reduction in retransmission directly impacts the latency of the system which can be seen from Figure 5. Figure 5 presents the average latency that a user can experience to complete the transmission with different penetration losses. It can be observed that the proposed algorithm results in significant latency reduction while achieving the improve information rate. For example, at the penetration loss of 20 $\mathrm{dB}$, the average latency of multi-cell with RRS is $12.4 \mathrm{~ms}$, whereas, the latency of the system reduced to $11.1 \mathrm{~ms}$ with the proposed algorithm. This results in approx. 10.4\% reduction in average latency compared to RRS.

\section{CONCLUSion}

In this paper, we have presented an interference-aware resource allocation algorithm to improve the overall system link reliability and latency at the same time to enable various URLLC use-case scenarios. The results reveal that inter-cell interference is the dominant factor in degrading the link quality, which leads to more retransmissions and eventually increases the system latency. The proposed algorithm aims to address the inter-cell interference problem and it has been shown that the proposed algorithm achieves considerable gains compared to traditional round-robin scheduling. The results show that the proposed algorithm is able to achieve simultaneous $7 \%$ gain in information rate and $10 \%$ in latency compared to traditional schemes.

\section{ACKNOWLEDGEMENT}

"This project has received funding from the European Unions Horizon 2020 research and innovation program under grant agreement No 668995. This material reflects only the authors view and the EC Research Executive Agency is not responsible for any use that may be made of the information it contains." 


\section{REFERENCES}

[1] "Framework and overall objectives of the future development of IMT for 2020 and beyond," document ITU-R, Recommendation M.2083, Tech. Rep., 2015.

[2] M. I. Ashraf, C.-F. Liu, M. Bennis, and W. Saad, "Towards low-latency and ultra-reliable vehicle-to-vehicle communication," in European Conference on Networks and Communications (EuCNC), 2017, pp. 1-5.

[3] I. Ahmad, T. Kumar, M. Liyanage, M. Ylianttila, T. Koskela, T. Braysy, A. Anttonen, V. Pentikinen, J.-P. Soininen, and J. Huusko, "Towards gadget-free internet services: A roadmap of the Naked world," Telematics and Informatics, vol. 35, no. 1, pp. 82 - 92, 2018.

[4] H. Shariatmadari, Z. Li, M. A. Uusitalo, S. Iraji, and R. Jntti, "Link adaptation design for ultra-reliable communications," in IEEE International Conference on Communications (ICC), 2016, pp. 1-5.

[5] "Study on new radio access technology physical layer aspects," document 3GPP, TR 38.802 V14.2.0, Tech. Rep., 2017.

[6] M. M. Alam, H. Malik, M. I. Khan, T. Pardy, A. Kuusik, and Y. L. Moullec, "A Survey on the Roles of Communication Technologies in IoT-Based Personalized Healthcare Applications," IEEE Access, vol. 6, pp. 36611-36631, 2018.

[7] V. Hytönen, Z. Li, B. Soret, and V. Nurmela, "Coordinated multi-cell resource allocation for $5 \mathrm{~g}$ ultra-reliable low latency communications," in European Conference on Networks and Communications (EuCNC), 2017, pp. 1-5.

[8] G. Pocovi, M. Lauridsen, B. Soret, K. I. Pedersen, and P. Mogensen, "Ultra-reliable communications in failure-prone realistic networks," in International Symposium on Wireless Communication Systems (ISWCS), 2016, pp. 414-418.

[9] C. She, C. Yang, and T. Q. S. Quek, "Radio resource management for ultra-reliable and low-latency communications," IEEE Communications Magazine, vol. 55, no. 6, pp. 72-78, 2017.

[10] "Introduction of NB-IoT ", document 36.331-RP-161248 3GPP TSGRAN Meeting 72, Ericsson, Nokia, ZTE, NTT DOCOMO Inc., Busan, South Korea, Tech. Rep., 2016.

[11] H. Malik, N. Kandler, M. M. Alam, I. Annus, Y. L. Moullec, and A. Kuusik, "Evaluation of low power wide area network technologies for smart urban drainage systems," in 2018 IEEE International Conference on Environmental Engineering (EE), 2018, pp. 1-5.

[12] H. Pervaiz, Q. Ni, and C. C. Zarakovitis, "User adaptive QoS aware selection method for cooperative heterogeneous wireless systems: A dynamic contextual approach," Future Generation Computer Systems, vol. 39 , pp. $75-87,2014$, special Issue on Ubiquitous Computing and Future Communication Systems.

[13] H. Malik, H. Pervaiz, M. M. Alam, Y. L. Moullec, A. Kuusik, and M. A Imran, "Radio Resource Management Scheme in NB-IoT Systems," IEEE Access, vol. 6, pp. 15051-15 064, 2018.

[14] "Cellular System Support for Ultra Low Complexity and Low Throughput Internet of Things ," document 3GPP TR 45.820 v13.0.0, Tech. Rep., 2015.

[15] "NB IoT Capacity evaluation," document 3GPP TR1-157248, TSG RAN1 Meeting 83, Tech. Rep., 2015. 